\title{
Fabrication and Evaluation of a Xenogeneic Decellularized Nerve-Derived Material: Preclinical Studies of a New Strategy for Nerve Repair
}

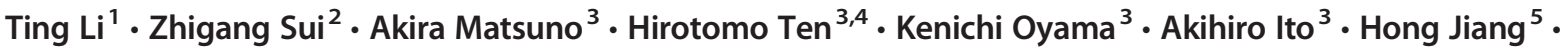 \\ Xiaomin Ren $^{5} \cdot$ Rabia Javed $^{1} \cdot$ Lihua Zhang $^{2} \cdot$ Qiang $\mathrm{Ao}^{1,6}$ (I)
}

Published online: 22 November 2019

(C) The American Society for Experimental NeuroTherapeutics, Inc. 2019

\begin{abstract}
The repair and regeneration of transected peripheral nerves is an important area of clinical research, and the adhesion of anastomosis sites to surrounding tissues is a vital factor affecting the quality of nerve recovery after nerve anastomosis. This study involves the generation of a novel nerve repair membrane derived from decellularized porcine nerves using a unique, innovative technique. The decellularized nerve matrix was verified to be effective in eliminating cellular components, and it still retained some neural extracellular matrix components and bioactive molecules (collagens, glycosaminoglycans, laminin, fibronectin, TGF- $\beta$, etc.), which were mainly determined by proteomic analysis, histochemistry, immunohistochemistry, and enzyme-linked immunosorbent assay. Cytotoxicity, intracutaneous reactivity, hemolysis, and cell affinity analyses were conducted to confirm the biosecurity of the nerve repair membrane. The in vivo functionality was assessed in a rat sciatic nerve transection model, and indices of functional nerve recovery, including the measurement of the claw-spread reflex, nerve anastomosis site adhesion, electrophysiological properties, and the number of regenerated nerve fibers, were evaluated. The results indicated that the nerve repair membrane could effectively prevent adhesion between the nerve anastomosis sites and the surrounding tissues and enhance nerve regeneration, which could be attributed to its various bioactive components. In conclusion, the novel nerve repair membrane derived from xenogeneic decellularized nerves described in this study shows great potential auxiliary clinical treatment for peripheral nerve injuries.
\end{abstract}

Key Words Nerve repairmembrane $\cdot$ xenogeneic decellularized matrix $\cdot$ proteomics $\cdot$ anti-adhesion $\cdot$ peripheral nerveregeneration

\section{Introduction}

Peripheral nerve injury is a common clinical disease that affects $2.8 \%$ of trauma patients globally [1]. It affects the quality of life of more than 1 million people each year worldwide and causes a huge medical and economic burden for society [2]. Despite advancements in microsurgical techniques, the

Lihua Zhang

lihuazhang@dicp.ac.cn

Qiang Ao

aoqiang@tsinghua.edu.cn

1 Department of Tissue Engineering, China Medical University, Shenyang, China

2 Key Laboratory of Separation Science for Analytical Chemistry, National Chromatographic Research and Analysis Center, Dalian Institute of Chemical Physics, Chinese Academy of Sciences, Dalian, China reconstruction of transected peripheral nerves remains a formidable challenge in reconstructive surgery. At present, the main treatment for transected peripheral nerves is direct endto-end suturing of the injured nerve ends. However, direct nerve anastomosis is still far from ideal because of the invasion of surrounding tissue into the anastomosis site, resulting in adhesion and painful neuroma formation [3-7]. Therefore,

3 Department of Neurosurgery, Teikyo University School of Medicine, Tokyo, Japan

4 Department of Judo Physical Therapy, Faculty of Health, Teikyo Heisei University, Tokyo, Japan

5 Shandong Junxiu Biotechnology Company, Limited, Yantai, China

6 Institute of Regulatory Science for Medical Devices, Engineering Research Center in Biomaterials, Sichuan University, Chengdu, China 
developing a nerve repair membrane for anastomosis site is necessary to prevent connective tissue invasion into anastomosis site and promote nerve regeneration.

In the past few years, some materials have been created to prevent anastomosis site adhesion after nerve anastomosis [8-10]. These materials have included poly(DL-lactic acid) film [8], chitosan film [9], and collagen wrapper [10]. Although they had an anti-adhesion effect, they were associated with some shortcomings. The former 2 materials had poor biocompatibility and a long degradation period, and none of the 3 materials contained various bioactive molecules. Hence, it was urgent to create a new nerve wrap that prevented adhesion and promoted nerve regeneration after anastomosis. The evidence suggests that extracellular matrix (ECM) materials, especially those derived from site-specific tissues, are more useful as reconstructive tissues than synthetic and nonspecific tissue-derived materials [11-13]. Zilic et al. [14] demonstrated that the porcine nerve was very similar to the human nerve in terms of its anatomical, biochemical, and cellular components. Porcine decellularized nerves without cellular components did not elicit an immune response [15]. Meanwhile, decellularized porcine nerves retain different kinds of functional proteins, including collagens, glycosaminoglycans, proteoglycans, and growth factors, which might provide a native-like microenvironment that would favor Schwann cell proliferation and axon growth [14, 16-18]. Therefore, porcine decellularized nerves would be an ideal nerve repair material. Recent studies have found that Schwann cells cultured in contact with decellularized porcine nerves could survive and proliferate and retain their own phenotype [16], and evidence has suggested that decellularized porcine nerve-derived hydrogels could enhance the wrapping of Schwann cells around areas of neuritis and promote axon growth in vitro [19]. In an animal experiment, porcine decellularized nerve-derived hydrogels containing conduits were integrated into transected sciatic nerves of rats, and better axonal regeneration was achieved in comparison to the results of using hollow conduits [20]. Therefore, both in vitro and in vivo studies have proven that porcine decellularized nerves show excellent performance in nerve regeneration.

In the current study, a novel nerve repair membrane derived from decellularized porcine nerves was successfully manufactured to prevent the adhesion of nerve anastomosis sites to surrounding tissues and to support nerve regeneration. To determine the components of the porcine decellularized nerve matrix, high-performance and high-resolution proteomic analysis, dimethylmethylene blue assay, hydroxyproline assay, Picrosirius red staining, immunohistochemistry, and enzyme-linked immunosorbent assay were performed. The biocompatibility of the nerve repair membrane was evaluated by cytotoxicity assay, intracutaneous reactivity test, hemolysis test, and cell affinity analysis. Furthermore, its treatment efficacy in transected sciatic nerves in a rat model was determined through claw-spread reflex test, observations of anastomosis site tissue adhesion, electrophysiological assay, and histological analysis of the regenerated nerve fibers.

\section{Materials and Methods}

\section{Manufacture of the Porcine Decellularized Nerve-Derived Nerve Repair Membrane}

Preparation of Porcine Decellularized Nerve Matrix Fresh sciatic nerves were harvested from the lower limbs of crossbred pigs obtained from a market according to ISO 22442-2, and the nerves were cut into $5-\mathrm{mm}$ pieces prior to decellularization. Briefly, the nerves were agitated and rinsed with deionized water for $6 \mathrm{~h}$ at room temperature. Then, the nerves were subjected to chemical decellularization in $3.0 \%$ Triton X-100 (Sigma, USA) for $12 \mathrm{~h}$ and washed with deionized water (3 times for $15 \mathrm{~min}$ each), 4.0\% sodium deoxycholate (Sigma, USA) for $24 \mathrm{~h}$, and deionized water again (3 times for 15 min each) with constant vibration. The resulting decellularized nerve matrix was lyophilized and stored until use. Hematoxylin and eosin (HE) staining and residual DNA content test were performed to analyze the efficiency of the decellularization of the porcine nerves. The TIANamp Genomic DNA Kit (TIANGEN, China) was used to isolate the genomic DNA from fresh nerves and decellularized nerve matrix. Later, the isolated DNA was analyzed and quantified using agarose gel electrophoresis and a NanoDrop spectrophotometer.

Manufacture of the Nerve Repair Membrane The lyophilized porcine decellularized nerve matrix was transformed into a powder using a tissue homogenizer (SPEX SamplePrep, USA). Then, the powder was solubilized in $0.3 \%$ acetic acid with constant vibration for $24 \mathrm{~h}$. After solubilization was complete, the digested solution was transferred to a plate, and the solution-to-membrane transition of the decellularized nerve matrix was induced by drying at room temperature. Finally, the $\mathrm{pH}$ was adjusted to 7.4 with $0.1 \mathrm{~N} \mathrm{NaOH}$, and the nerve repair membrane was washed with deionized water 3 times.

\section{Composition Analysis}

Proteomic Analysis Mass spectrometry was used to analyze the protein contents of the nerve repair membrane, decellularized nerve matrix, and fresh nerves. The samples were prepared using imFASP method with slight modification [21]. Protein extract was determined by using a BCA kit (Beyotime Biotechnology, China). The proteins in each group were digested with trypsin (enzyme to protein ratio of 1:30), and then $1 \mu \mathrm{g}$ of each protein extract was analyzed by a nanoRPLC-ESI-MS/MS system consisting of an EasyNano 
HPLC system and a Q-Exactive MS (Thermo Fisher, USA). The nano-RPLC separation was performed using a capillary column that was packed in-house $(0.075 \mathrm{~mm}$ id $\times 140 \mathrm{~mm})$ (Sino Sumtech, China) with C18 silica particles ( $5 \mu \mathrm{m}, 100 \AA$ ) (Thermo Electron, USA). Mobile phase A ( $0.1 \%$ formic acid in $\left.\mathrm{H}_{2} \mathrm{O}\right)$ and $\mathrm{B}(0.1 \%$ formic acid in acetonitrile) were used to generate an 80-min gradient that was comprised of $5 \mathrm{~min}$ of $2 \%$ B followed by 65 min of 5 to $50 \%$ B, with a final step of $80 \%$ B for $10 \mathrm{~min}$; the flow rate was $200 \mathrm{nl} / \mathrm{min}$.

The mass spectrometer was operated in positive mode with a spray voltage of $2.25 \mathrm{kV}$ and a temperature in the ion transfer capillary of $200{ }^{\circ} \mathrm{C}$. The MS/MS collision energy was set at $28 \%$, and all MS/MS spectra were acquired in data-dependent mode. A full MS scan was acquired from $\mathrm{m} / \mathrm{z}, 300$ to $\mathrm{m} / \mathrm{z}, 1800$ and was followed by 10 data-dependent MS/MS events. The MS/MS scans were performed at a resolution of 17,500 (AGC, $1 \mathrm{E}^{5}$; maximum injection time, $60 \mathrm{~ms}$ ), and the data were acquired in centroid mode using a 20 -s exclusion window. The unassigned ions or those with a charge of +1 or $>+7$ were rejected.

The raw data were uploaded into Proteome Discoverer (PD, version 1.4.1.14) with Mascot (2.3.2) and were searched against the UniProt Knowledgebase (UniProtKB) complete pig proteome sequence database (release 2018_10, 48,969 entries). The mass tolerances were set at $0.5 \mathrm{Da}$ for the parent ions and at $10 \mathrm{ppm}$ for the fragments. The minimal peptide length was 6 amino acids. Carbamidomethylation $(\mathrm{C},+$ $57.0215 \mathrm{Da}$ ) was used as the fixed modification. Oxidation $(\mathrm{M},+15.9949 \mathrm{Da})$ and acetylation (protein $\mathrm{N}$ termini, + 42.0106 Da) were searched as variable modifications. The peptide and protein identifications were filtered by PD with false discovery rate $\leq 1 \%$. At least 1 unique peptide was required for the identification of each protein. The percentage of ECM proteins was calculated by determining the relative abundances of all the identified ECM proteins, and the relative abundances of the identified proteins were expressed as $\log _{10}$ values.

Glycosaminoglycans Analysis The glycosaminoglycans (GAGs) content of the decellularized nerve matrix and fresh nerves was detected and quantified by dimethylmethylene blue assay (GenMed Scientific INC, USA) according to the manufacturer's instructions.

Hydroxyproline Assay A hydroxyproline assay was carried out to determine the total collagen in the decellularized nerve matrix and fresh nerves. This assay utilizes the formation of a chromophore by the oxidation of hydroxyproline with chloramine-T (sodium $N$-chloro- $p$ toluenesulfonamide), which can be measured at $570 \mathrm{~nm}$ [22]. The total collagen content was calculated according to the ratio of hydroxyproline to collagen, which was 1:7.69 $[16,23]$.
Picrosirius Red Staining Picrosirius red staining was performed to detect collagen I and collagen III in decellularized nerve matrix and fresh nerves. The samples were fixed in $4 \%$ paraformaldehyde (PFA) for $24 \mathrm{~h}$ followed by several washes in phosphate-buffered saline (PBS, $\mathrm{pH}$ 7.4) for $2 \mathrm{~h}$. Then, the fixed samples were dehydrated with a graded ethanol series, embedded in paraffin wax, sectioned into 5 - $\mu \mathrm{m}$ sections, and affixed to microscope slides. The slides were stained with Picrosirius red to visualize collagen I and collagen III distribution under visible light and polarized illumination (BX53; Olympus, Japan).

Immunohistochemistry For immunohistochemistry (IHC), the slides of nerve tissues were pretreated with citrate buffer at 95 ${ }^{\circ} \mathrm{C}$ for antigen retrieval. Next, the UltraSensitive SP (Mouse/ Rabbit) IHC Kit (MXB® Biotechnologies, China) was used according to the manufacturer's instructions. The sections were incubated in $3 \%(\mathrm{v} / \mathrm{v}) \mathrm{H}_{2} \mathrm{O}_{2}$ for 10 min to block endogenous peroxidase activity. Nonspecific staining was further blocked with nonimmune serum at $37{ }^{\circ} \mathrm{C}$ for $30 \mathrm{~min}$. Anticollagen IV antibody, anti-laminin antibody, and antifibronectin antibody (Abcam, USA) were used at a 1:200 dilution for $12 \mathrm{~h}$ at $4{ }^{\circ} \mathrm{C}$. The secondary antibody was used according to the instructions from the UltraSensitive SP (Mouse/Rabbit) IHC Kit. Subsequently, the peroxidase reaction was visualized with a DAB Peroxidase Substrate Kit (MXB® Biotechnologies, China). After counterstaining with hematoxylin, the slides were dehydrated, cleared, mounted, and visualized with an Olympus BX53 (Japan) light microscope.

Enzyme-Linked Immunosorbent Assay An enzyme-linked immunosorbent assay (ELISA) was carried out to detect collagen I, collagen IV, laminin (LN), and fibronectin (FN) in protein extracts from decellularized nerve matrix and fresh nerves. ELISA kits (USCN Life Science Technology, China) were used to analyze the protein contents according to the manufacturer's instructions. Standard samples and protein extracts of decellularized nerve matrix and fresh nerves $(100 \mu \mathrm{l})$ were added to the wells in triplicate and incubated for $2 \mathrm{~h}$ at room temperature. The color reaction was monitored at $405 \mathrm{~nm}$, and the data from the standard samples were plotted as the background-corrected absorbance (OD) versus the concentration. The contents of collagen I, collagen IV, LN, and FN in the 2 groups were calculated based on the appropriate standard curve.

\section{Biocompatibility of the Nerve Repair Membrane}

Cytotoxicity Assay The cytotoxicity of the nerve repair membrane was investigated by 3-(4,5-dimethylthiazol-2-yl)-2,5diphenyltetrazolium bromide (MTT) assay. Schwann cells (RSC96) were cultured in complete Dulbecco's modified 
Eagle medium (DMEM) with 10\% fetal bovine serum and $1 \%$ penicillin/streptomycin, as described previously [24]. The protocols were performed as described in ISO 10993-5. The conditioned media were obtained by incubating $0.2 \mathrm{~g}$ nerve repair membrane in $1 \mathrm{ml} \mathrm{DMEM}$ at $37{ }^{\circ} \mathrm{C}$ for $24 \mathrm{~h}$ and then collected by filtering the remains. In addition, a normal medium control (DMEM), a negative control (polyethylene), and a positive control (DMSO) were prepared. The cell pellets were counted and then seeded at a density of $2 \times 10^{4}$ cells/well in 96-well plates. Then, the 96-well plates were transferred to an incubator at $37{ }^{\circ} \mathrm{C}$ with $5 \% \mathrm{CO}_{2}$ and $95 \%$ humidity. After 3 days of culture, the culture medium of the Schwann cells was replaced with $25 \mu \mathrm{l}$ MTT ( $5 \mathrm{mg} / \mathrm{ml})$, which was followed by incubation for $4 \mathrm{~h}$ at $37^{\circ} \mathrm{C}$. Then, the reaction solution was carefully removed from the 96-well plates, and $100 \mu \mathrm{l}$ of DMSO was added to dissolve the blue formazan product, after which the $\mathrm{OD}$ at $570 \mathrm{~nm}$ was measured. The cell viability (\%) was calculated according to the formula $\mathrm{OD}_{\text {test }} / \mathrm{OD}_{\text {medium }} \times$ 100 , where $\mathrm{OD}_{\text {test }}$ is the absorbance of each group and $\mathrm{OD}_{\text {medium }}$ is the absorbance of DMEM group. Six replicates for each group were used to assess cell viability.

\section{Scanning Electron Microscopy Observation of Cell Affinity} Scanning electron microscopy (SEM) was used to observe Schwann cells cultured on the surface of the nerve repair membrane. Briefly, Schwann cells were seeded on the nerve repair membrane surface and incubated in DMEM with $10 \%$ fetal bovine serum and $1 \%$ penicillin/streptomycin. After 3 days of culture, the samples were coated with gold and observed at an acceleration voltage of $25 \mathrm{kV}$ by SEM (S-4800; Hitachi, Japan).

Intracutaneous Reactivity Test The intracutaneous reactivity test was performed according to ISO 10993:2010 (Biological Evaluation of Medical Devices-Part 10). The subjects were male New Zealand White rabbits $(n=3,2.5 \pm 0.2 \mathrm{~kg})$ that were kept in an animal facility under standard housing conditions. The rabbits were maintained under a 12-h light/dark cycle and had free access to water and food. The room temperature $\left(22.0 \pm 3.0^{\circ} \mathrm{C}\right)$ and ambient humidity $(50 \pm 20 \%)$ were monitored daily. Before treatment, the fur on the back and both sides of the spine of each rabbit was sheared to obtain a sufficiently large injection area. Then, the injection area was disinfected with a $75 \%$ alcohol-soaked gauze pad. The extraction solution for the nerve repair membrane $(0.2 \mathrm{~g} / \mathrm{ml})$ was prepared in physiological saline at $37^{\circ} \mathrm{C}$ for $24 \mathrm{~h}$ and then collected by filtering the remains. A volume of $0.2 \mathrm{ml}$ of extraction solution was injected intracutaneously into 10 separate sites on the left side of each rabbit spine, and the same dose of physiological saline was injected into sites on the corresponding right side of the spine. The injections were spaced approximately $1.0 \mathrm{~cm}$ apart. Then, observations of the injection sites were made immediately after injection as well as 24,48 , and $72 \mathrm{~h}$ after injection. The appearance of each site was evaluated by grading for erythema and edema, as per the following criteria: 0 , negligible; 1 , very slight; 2 , welldefined; 3 , moderate; 4 , severe. The mean scores of erythema and edema individually for the test and control sites were calculated. If the difference between the mean scores of the test sites and the control sites was less than or equal to 1.0 , it was considered to indicate an absence of intracutaneous irritation.

Hemolysis Test The hemolysis test was performed by a colorimetric assay that measured the release of cyanmethemoglobin in solution. A $2 \%(\mathrm{w} / \mathrm{v})$ red blood cell (RBC) suspension from Sprague-Dawley rats was prepared as described previously [25]. Briefly, rat blood was centrifuged at $4000 \mathrm{rpm} / \mathrm{min}$ and $4{ }^{\circ} \mathrm{C}$ for $15 \mathrm{~min}$, and the plasma was removed. Then, the cell pellets were resuspended in physiological saline for washing, followed by centrifugation as previously described. After washing 3 times, the cell pellets were resuspended in physiological saline at a final concentration of $2 \%(\mathrm{w} / \mathrm{v})$. The extraction solution of the nerve repair membrane $(0.2 \mathrm{~g} / \mathrm{ml})$ was prepared in physiological saline at $37^{\circ} \mathrm{C}$ for $24 \mathrm{~h}$. In addition, the same amount of physiological saline and deionized water were prepared as the negative and the positive control, respectively. Then, each solution was mixed with the RBC suspension and incubated for $1 \mathrm{~h}$ at $37^{\circ} \mathrm{C}$. Each solution was centrifuged at $3000 \mathrm{rpm} / \mathrm{min}$ for $10 \mathrm{~min}$, and the supernatant was measured at $\mathrm{OD}_{545}$. Later, the mean $\mathrm{OD}_{545}$ value and hemolytic rate were calculated.

\section{Functionality of the Nerve Repair Membrane In Vivo}

Surgery Procedure Male Sprague-Dawley rats $(n=32,220 \pm$ $10 \mathrm{~g}$ ) were used for in vivo nerve repair studies and kept in an animal room under standard housing conditions. The bilateral sciatic nerves of all animals were transected, followed by endto-end nerve anastomosis. The surgical operation was performed by the same surgeon under aseptic conditions with $2 \%$ pentobarbital sodium anesthesia $(25 \mathrm{mg} / \mathrm{kg})$ for all rats. An incision was made on the posterior-lateral side of the thigh, and the sciatic nerve was exposed carefully and cut quickly $0.5 \mathrm{~cm}$ above the bifurcation of the tibial and peroneal nerve with microsurgical scissors. The epineuria of the proximal and distal nerve ends were sutured using a 9-0 nylon microsuture. The right anastomosis sites were wrapped with the nerve repair membranes (as the treatment group), whereas the left anastomosis sites were not wrapped with nerve repair membranes (as the nontreatment group). The muscle and skin were closed with 6-0 nylon sutures.

The surgical procedure was performed strictly in accordance with the China Medical University guidelines for the ethical care of animals. All animals had free access to water 
and food. The spirit and appetites of the animals and the appearances of the surgical incisions were observed every day.

Claw-Spread Reflex test The claw-spread reflex was detected to assess the functional nerve recovery at 4,8 , and 12 weeks after surgery. The nerve functional recovery was graded as A, $\mathrm{B}$, or $\mathrm{C}$ according to the following scale: grade $\mathrm{A}$, rat responds to acupuncture and spreads its claws; grade $\mathrm{B}$, rat responds to acupuncture without spreading its claws; grade $\mathrm{C}$, rat does not respond to acupuncture.

Nerve Anastomosis Site Tissue Adhesion Observation Nerve anastomosis site adhesion to surrounding tissues was evaluated at 4,8 , and 12 weeks after surgery according to 3 grades: grade 0 , no adhesion; grade 1 , the anastomosis site was slightly adhered to the surrounding tissues but was able to be separated; grade 2, the anastomosis site was tightly adhered to the surrounding tissues and was difficult to separate.

Electrophysiological Evaluation Nerve functional recovery was also measured by electrophysiological assay at 4,8 , and 12 weeks after surgery. The rats were anesthetized with $2 \%$ pentobarbital sodium $(25 \mathrm{mg} / \mathrm{kg})$, and the nerve anastomosis site was re-exposed. Electrical stimuli were applied at the proximal and distal nerve trunks of the anastomosis site by a multichannel electrophysiological system (RM6240, China). The stimulation mode was set to pulse mode (stimulus intensity $=6 \mathrm{~mA}$; frequency $=1 \mathrm{~Hz}$; duration $=1 \mathrm{~ms})$. A recording electrode was inserted into an anterior tibial muscle to measure the compound muscle action potential (CMAP). The CMAP amplitude was recorded by measuring the vertical distance from the peak to the trough of the CMAP. The CMAP latencies induced by proximal and distal stimulation were recorded. The difference in the CMAP latencies was calculated, and the distance between the proximal and distal stimulation sites was measured to determine the motor nerve conduction velocity (MCV) across the nerve trunk between the proximal and distal stimulation points.

Toluidine Blue Staining of the Myelin Sheath After the electrophysiological assay, the nerve distal segments near the anastomosis site were harvested and fixed in $4 \%$ PFA for $48 \mathrm{~h}$, followed by washing in PBS. Then, the samples were embedded in Epon 812 epoxy resin and cut into $0.5-\mu \mathrm{m}$ semithin sections using glass knives. After being stained with toluidine blue, the sections were observed under a light microscope (BX53; Olympus, Japan), and 5 high power fields $(\mathrm{HPF}, \times 1000)$ were selected for each section (center, top, bottom, right, and left of the section). Then, the myelinated axon density (number of axons/HPF) was quantitatively determined using ImageJ.

Immunofluorescence of the Axon and Myelin Sheath For the immunofluorescence detection of the axon and myelin sheath, the distal nerve segments near the anastomosis site were fixed

a

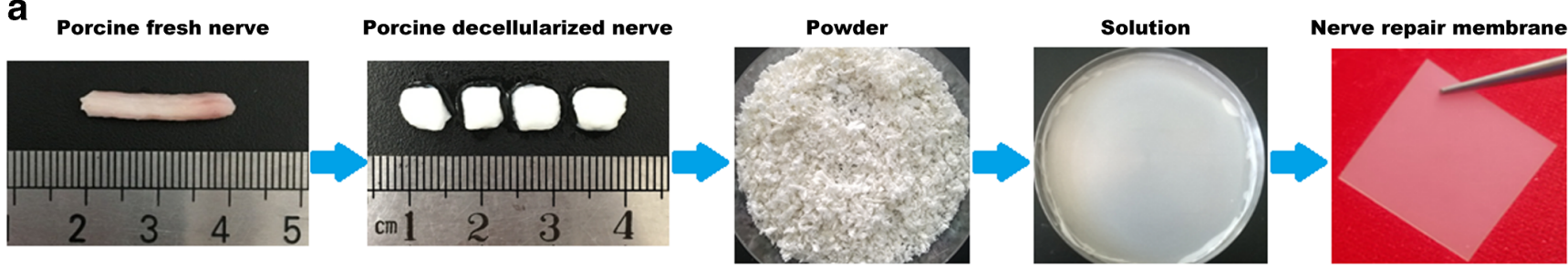

b
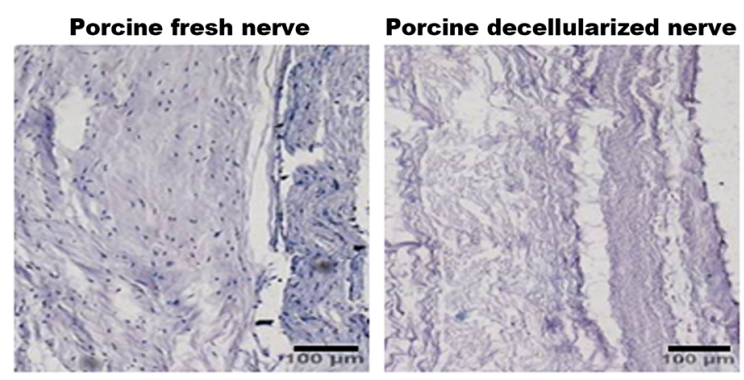

Fig. 1 Manufacturing procedure used for the nerve repair membrane (a) and the decellularization efficiency evaluation (b, c). (a) Porcine sciatic nerves were decellularized by chemical extraction, followed by tissue homogenization, acid digestion, and room temperature drying, resulting in the formation of the nerve repair membrane. (b) HE staining of fresh nerve and decellularized nerve tissue. Scale bar $=100 \mu \mathrm{m}$. (c) Agarose
C Agarose gel electrophoresis
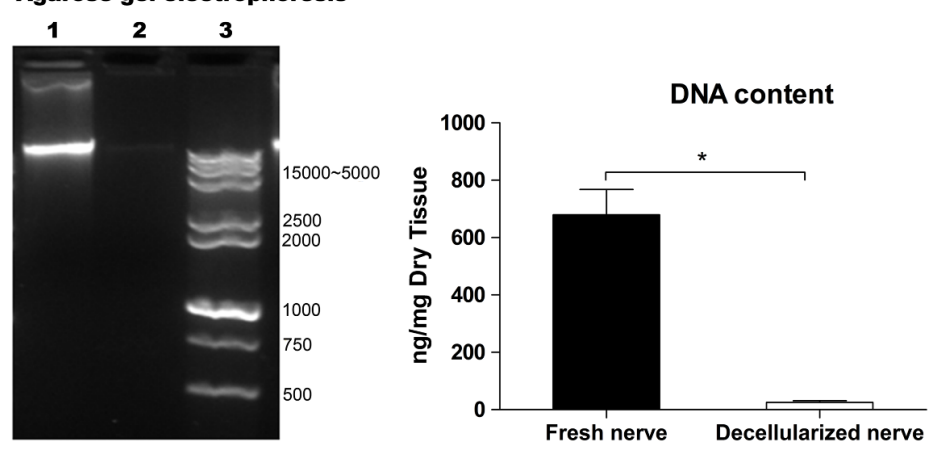

gel electrophoresis: lane 1 shows the DNA isolated from fresh nerve tissue; lane 2 shows DNA isolated from the decellularized nerve matrix; lane 3 shows the DNA marker (bp). DNA content: experiments were performed in triplicate, and data are expressed as the mean \pm S.D., $* p<$ 0.05 
a

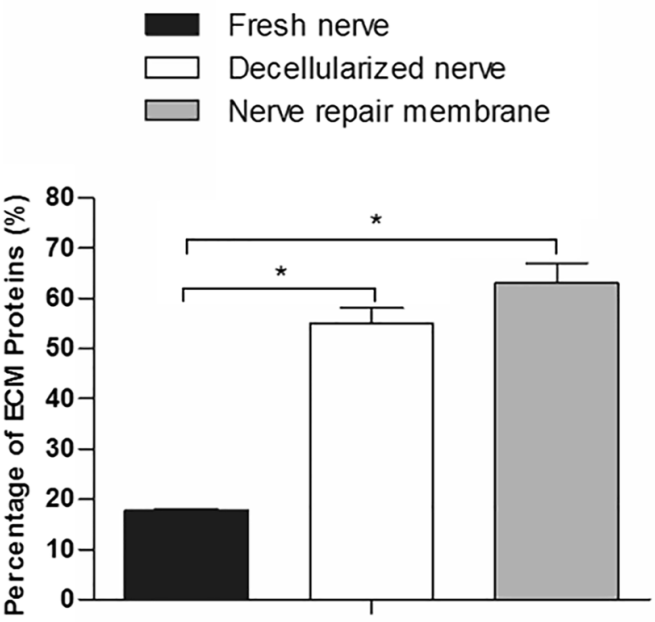

b

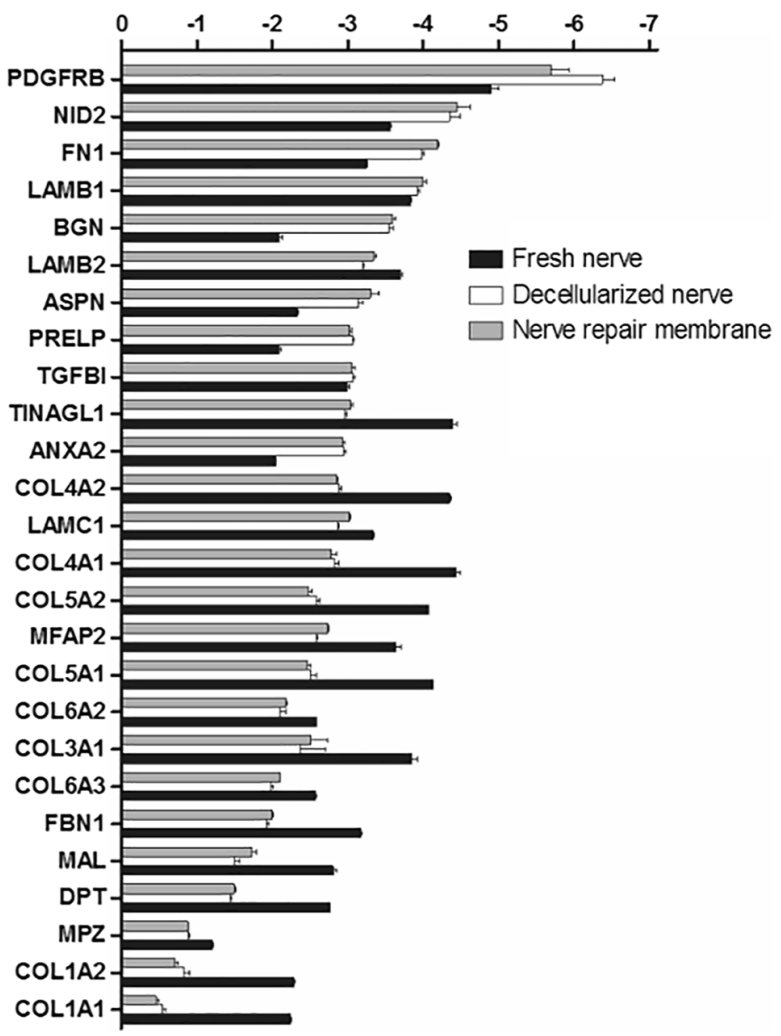

Fig. 2 Proteomic analysis of the nerve repair membrane, decellularized nerve matrix, and fresh nerve tissue. The percentage of identified ECM protein mass in the total protein mass from fresh nerves, decellularized nerve, and nerve repair membrane (a). The relative abundances of the identified proteins in fresh nerve tissue, decellularized nerve matrix, and nerve repair membrane determined by proteomic analysis were expressed as $\log _{10}$ values (b). COL1A1 = collagen I $\alpha 1$ chain; COL4A1 $=$ collagen IV $\alpha 1$ chain; $\mathrm{MPZ}=$ myelin protein zero; $\mathrm{DPT}=$ dermatopontin; $\mathrm{MAL}=$ structural constituent of myelin sheath; FBN1 = fibrillin-1; COL6A3 = collagen VI $\alpha 3$ chain; COL3A1 = collagen III $\alpha 1$ chain; COL6A2 $=$ collagen VI $\alpha 2$ chain; COL5A1 = collagen V $\alpha 1$ chain; MFAP2 = microfibrillar-associated protein 2 ; COL5A2 = collagen $\mathrm{V} \alpha 2$ chain; COL4A $1=$ collagen IV $\alpha 1$ chain; LAMC1 = laminin $\gamma 1$ subunit; COL4A2 = collagen IV $\alpha 2$ chain; ANXA2 = annexin A2; TINAGL1 = tubulointerstitial nephritis antigen-like $1 ; \mathrm{TGFBI}=$ transforming growth factor-beta-induced protein Ig-h3; PRELP = prolargin; ASPN = asporin; LAMB2 = laminin $\beta 2$ subunit; $\mathrm{BGN}=$ biglycan; LAMB1 $=$ laminin $\beta 1$ subunit; FN1 = fibronectin; NID2 = nidogen-2; PDGFRB = plateletderived growth factor receptor beta. The protein extracts were analyzed in triplicate, and the data are expressed as the mean \pm S.D., ${ }^{*} p<0.05$ in $4 \%$ PFA for $24 \mathrm{~h}$ at $4{ }^{\circ} \mathrm{C}$ and soaked in $30 \%$ sucrose solution for $6 \mathrm{~h}$ at $4{ }^{\circ} \mathrm{C}$. Histological sections $(10 \mu \mathrm{m})$ were prepared using a freezing microtome. The sections were incubated in $1 \%$ normal goat serum in PBS for $1 \mathrm{~h}$ at $24{ }^{\circ} \mathrm{C}$ and then treated with anti-neurofilament antibody (anti-NF200, 1:1000 dilution; Abcam, USA) and anti-S100 antibody (1:2000 dilution; Abcam, USA) for $12 \mathrm{~h}$ at $4{ }^{\circ} \mathrm{C}$. Following 3 to 4 washes in PBS, secondary antibody labeling was performed with goat anti-rabbit IgG antibody and goat anti-mouse IgG antibody at a 1:500 dilution (Abcam, USA). After counterstaining the cell nuclei with 2-(4-amidinophenyl)-6indolecarbamidine (DAPI), the sections were visualized by
Fig. 3 GAGs (a) and total collagen (b) contents of decellularized nerve matrix and fresh nerve tissue. Three samples of decellularized nerve matrix and fresh nerve tissue were assayed 3 times. Data are expressed as the mean \pm S.D., ${ }^{*} p<0.05$
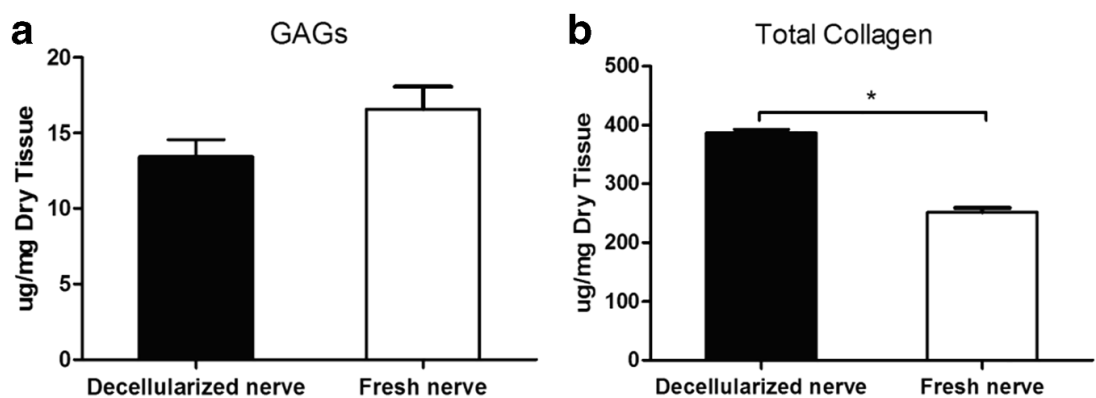

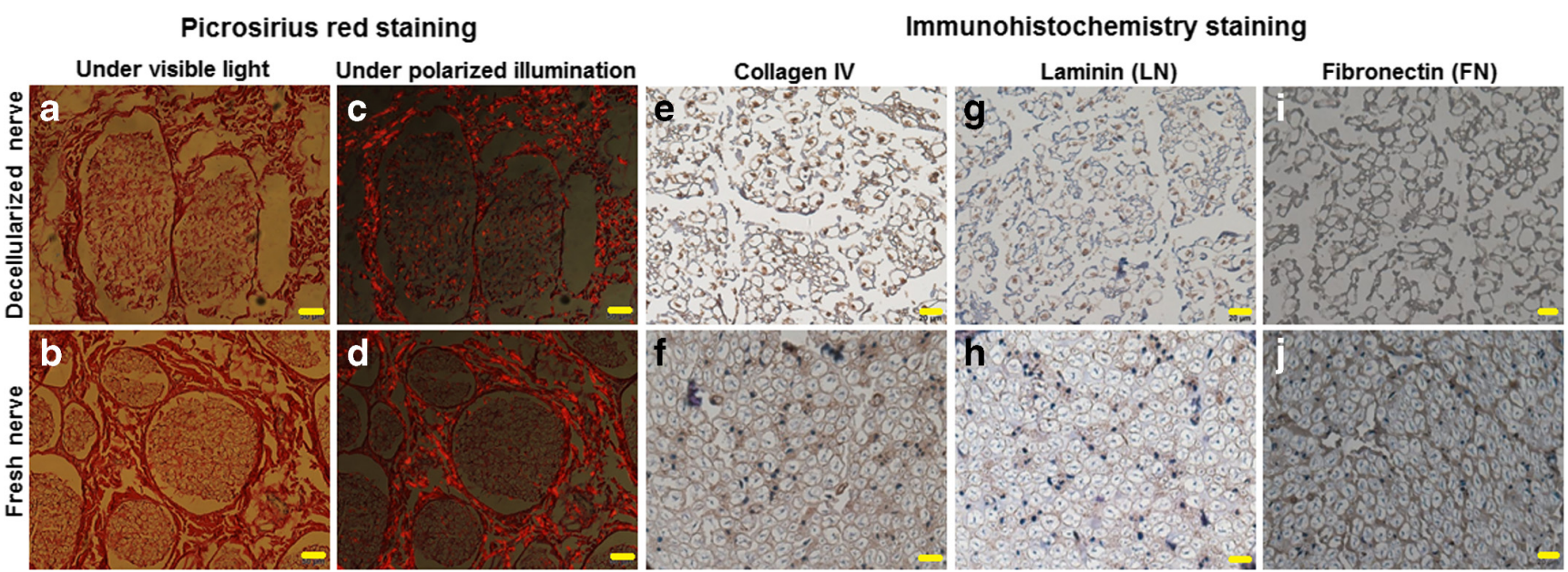

Fig. 4 Representative sections of decellularized nerve matrix and fresh nerve with labeling of collagen I, collagen IV, LN, or FN. For Picrosirius red staining (a-d), sections of decellularized nerve $(a, c)$ and fresh nerve tissue $(b, d)$ were viewed under visible light $(a, b)$ and polarized

laser scanning confocal microscopy (FV1200; Olympus, Japan).

\section{Statistical Analysis}

All data were expressed as the mean \pm standard deviation (S.D.) and were statistically analyzed using GraphPad Prism 5.0 software (USA). One-way analysis of variance (ANOVA) was used for the proteomic analysis; the Wilcoxon test was used for the claw-spread reflex analysis and the anastomosis site tissue adhesion evaluation, and the other data were statistically analyzed using $t$ tests. $p<0.05$ was considered statistically significant. Asterisks indicate a statistically significant difference between 2 groups.

\section{Results}

\section{Decellularization Efficiency Analysis}

The decellularized nerves were derived from porcine sciatic nerves by chemical decellularization, and the manufacturing procedure used for the decellularized, nerve-derived nerve repair membranes is shown in Fig. 1a. After complete decellularization,

Table 1 Main components of the decellularized nerve matrix and fresh nerve tissue as determined by ELISA

\begin{tabular}{lll}
\hline Component & Decellularized nerve matrix & Fresh nerve tissue \\
\hline Collagen I (ng/mg) & $3.32 \times 10^{5} \pm 0.61$ & $2.19 \times 10^{5} \pm 0.09$ \\
Collagen IV (ng/mg) & $14.36 \pm 4.11$ & $19.66 \pm 2.33$ \\
LN (ng/mg) & $25.81 \pm 4.65^{*}$ & $45.25 \pm 2.96$ \\
FN (ng/mg) & $2.82 \pm 0.27^{*}$ & $28.99 \pm 2.87$ \\
\hline
\end{tabular}

$* p<0.05$ versus fresh nerve illumination $(c, d)$. Scale bar $=50 \mu \mathrm{m}$. For IHC staining $(e-j)$, sections of decellularized nerve $(e, g, i)$ and fresh nerve tissue $(f, h, j)$ were stained for collagen IV (e, f), LN (g, h), or FN (i, j). Scale bar $=20 \mu \mathrm{m}$

the porcine sciatic nerves turned white and loosened, and HE staining confirmed that the decellularization process eliminated the cell nuclei from the fresh nerve tissue (Fig. 1b). The results of agarose gel electrophoresis revealed that there was almost no DNA in the decellularized nerve matrix, and the quantitative analysis showed that the DNA content decreased from 679.40 $\pm 44.22 \mathrm{ng} / \mathrm{mg}$ in dry tissue from fresh nerves to $25.38 \pm 3.13 \mathrm{ng} /$ $\mathrm{mg}$ in dry tissue from the decellularized nerve matrix $(p<0.05)$ (Fig. 1c). The residual DNA content $(<50 \mathrm{ng} / \mathrm{mg})$ of the decellularized nerve matrix was considered to meet the standard for transplantation [26, 27].

\section{Composition Analysis}

Proteomic Analysis of the Nerve Repair Membrane, Decellularized Nerve Matrix, and Fresh Nerve tissue The protein mass spectrometry was characterized by high performance and high resolution, so a nano-RPLC-ESI-MS/MS system was used to quantitatively analyze the protein components in the nerve repair membrane, decellularized nerve, and fresh nerve tissue. Fig. 2a shows the percentage of identified ECM protein mass in the total protein mass of each group. The percentage of identified ECM proteins in the nerve repair membrane (63.02 \pm $4.01 \%)$ was significantly higher than that in fresh nerve tissue $(17.78 \pm 0.19 \%)(p<0.05)$ and similar to that in decellularized nerve tissue $(54.94 \pm 3.17 \%)$. Several major proteins in the nerve tissue were selected for comparison among the 3 groups, which showed the quantitative changes in these major proteins throughout the process that started with fresh nerve tissue to generate the decellularized nerve matrix and ultimately the nerve repair membrane (Fig. $2 b$ ). The different changes in different proteins might be attributed to the decellularization processes, isoelectric points, hydrophilicity, and hydrophobicity. However, there were no significant differences in these proteins in the nerve repair 
Fig. 5 Cytotoxicity (a) and cell affinity (b, c) of the nerve repair membrane. The $\mathrm{OD}_{570}$ values of the 3 control groups and the nerve repair membrane group according to MTT assay (a). SEM micrographs of cultured Schwann cells on the nerve repair membrane surface at $\times 200$ magnification (b) and $\times 1000$ magnification $(c)$. The inset in the SEM micrograph (b) indicates the position of the image shown in (c)

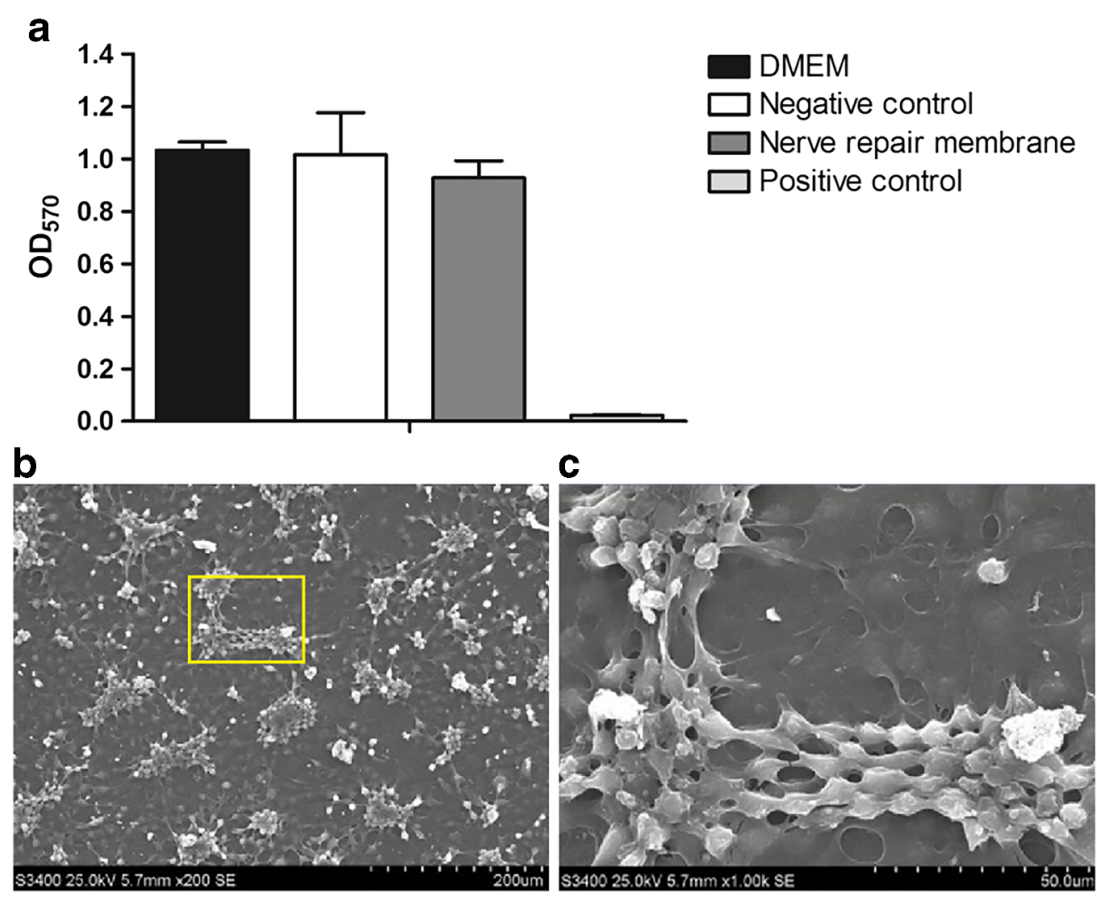

membrane and the decellularized nerve matrix, which indicated that the manufacturing process of the nerve repair membrane did not cause the loss of much protein.

The proteomic analysis results showed that not only major structural proteins, such as collagens, LN, and FN, but also some of the bioactive molecules that favor nerve regeneration (according to data from UniProtKB), such as annexin A2, transforming growth factor $\beta$, and nidogen-2, were detected in the decellularized nerve matrix and the nerve repair membrane.

\section{GAGs and Total Collagen Content in Decellularized Nerve} Matrix and Fresh Nerve Tissue As GAGs and collagen were the main components of ECM, the GAGs and collagen contents retained in the decellularized nerve matrix and fresh nerve tissue were determined. In the decellularized nerve matrix and fresh nerve tissue, the GAGs content was $13.4 \pm 1.1$ and $16.6 \pm 1.5 \mu \mathrm{g} / \mathrm{mg}$ of dry tissue, respectively (Fig. 3a). There was no difference in the GAGs content between the 2 groups. Moreover, the total collagen content was $386.9 \pm 5.9$ and $251.8 \pm 7.3 \mu \mathrm{g} / \mathrm{mg}$ of dry tissue, respectively $(p<0.05)$ (Fig. 3b).

\section{Collagen I, Collagen IV, LN, and FN in Decellularized Nerve} Matrix and Fresh Nerve Tissue Because collagen I, collagen IV, LN, and FN were the main components of ECM in porcine peripheral nerves, Picrosirius red staining, immunohistochemistry, and ELISA were performed to measure these proteins. As shown in Fig. 4, the staining of the decellularized nerve matrix and fresh nerve tissue with Picrosirius red revealed the presence of collagen I and collagen III (Fig. 4a-d); however, a large amount of collagen I (red) was found around the perineurium, and no collagen III (green) was detected. Immunohistochemical staining was used to detect collagen IV (Fig. 4e, f), LN (Fig. 4g, h), and FN (Fig. 4i, j), which are located around the endoneurium. The results demonstrated that collagen I, collagen IV, LN, and FN were retained in nerve tissues after decellularization. Furthermore, the quantitative detection of collagen I, collagen IV, LN, and FN was performed by ELISA (Table 1). The contents of collagen I, collagen IV, LN, and FN were $3.32 \times 10^{5}, 14.36,25.81$, and 2.82 $\mathrm{ng} / \mathrm{mg}$, respectively, in the decellularized nerve matrix and $2.19 \times 10^{5}, 19.66,45.25$, and $28.99 \mathrm{ng} / \mathrm{mg}$, respectively, in the fresh nerve tissue. The contents of LN and FN were significantly decreased in the decellularized nerve matrix compared to those in fresh nerve tissue $(p<0.05)$, although there was no difference in the amounts of collagen I and collagen IV between the 2 groups.

\section{Biocompatibility Evaluation of the Nerve Repair Membrane}

Cytotoxicity An MTT assay was performed to determine the cytotoxicity of the nerve repair membrane. After 3 days of cell culture, the $\mathrm{OD}_{570}$ value for the nerve repair membrane group was $0.928 \pm 0.065$, that for the DMEM group was $1.033 \pm$ 0.032 , that for the negative control group was $1.016 \pm 0.161$, and that for the positive control group was $0.023 \pm 0.003$ (Fig. $5 a)$. There was no significant difference in the $\mathrm{OD}_{570}$ value obtained for the nerve repair membrane group and the negative control group. The cell viability of the Schwann cells in the membrane group (89.83\%) was similar to that in the negative control group $(98.31 \%)$, but was significantly higher 


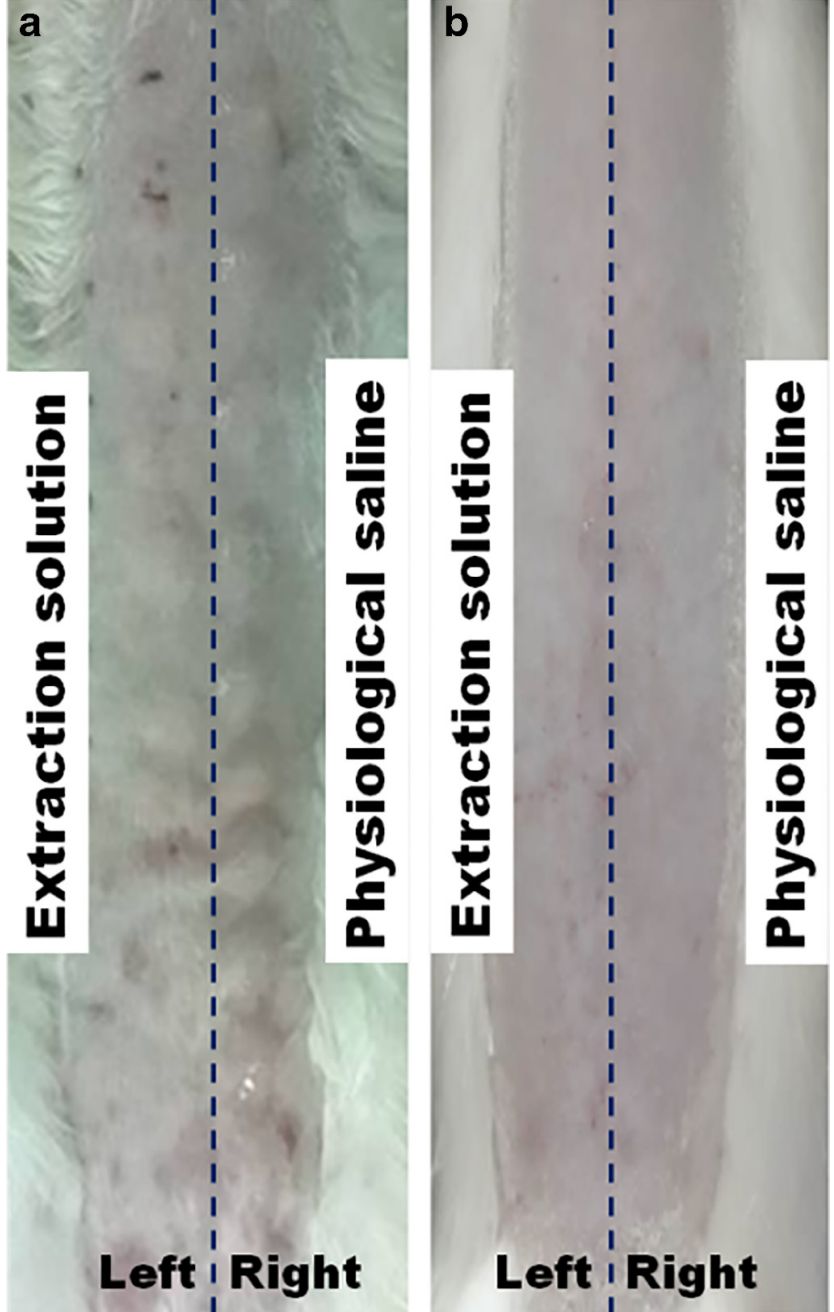

Fig. 6 Intracutaneous reactivity test of the nerve repair membrane extract. The left side of the rabbit spine was injected with extraction solution $(0.2$ $\mathrm{g} / \mathrm{ml}$ ) from the nerve repair membrane; the right side of the rabbit spine was injected with physiological saline. The dermal appearance (erythema and edema) of rabbits was observed immediately after injection (a) and at $72 \mathrm{~h}$ after injection (b)

than that in the positive control group $(2.23 \%)(p<0.05)$. These results indicated that the nerve repair membrane produced by our process showed no toxicity in cells.

SEM Observation of Cell Affinity Scanning electron micrographs showed that Schwann cells maintained good cell morphology with elongated processes and a high number of cell-

Table 2 Mean $\mathrm{OD}_{545}$ value and $\mathrm{HR}$ according to the hemolysis assay

\begin{tabular}{lll}
\hline Group & $\mathrm{OD}_{545}$ & HR (\%) \\
\hline Nerve repair membrane & $0.0016 \pm 0.013$ & 1.07 \\
Physiological saline & $-0.0066 \pm 0.027$ & 0 \\
Deionized water & $0.7555 \pm 0.053$ & 100 \\
\hline
\end{tabular}

cell contacts on the nerve repair membrane surface (Fig. $5 \mathrm{~b}, \mathrm{c})$. The results showed that the nerve repair membrane could support cell attachment and proliferation.

Intracutaneous Reactivity Test All rabbits appeared clinically normal during the test period. Dermal observations of erythema and edema were made at 24,48 , and $72 \mathrm{~h}$ after injection. Fig. 6 presents the dermal appearance of the rabbits immediately after (a) and $72 \mathrm{~h}$ after injection (b). All of the mean scores for the physiological saline sites and the membrane extract sites were 0 , which signifies that all injection sites showed no erythema or edema until $72 \mathrm{~h}$ after injection. The results showed that there was no significant irritation caused by the membrane extract in rabbits after intradermal injection.

Hemolysis Test The hemolysis test was performed to evaluate the blood compatibility of the nerve repair membrane, and the hemolytic rate (HR) represented the extent of the fracture and dissolution of RBCs caused by contact of the nerve repair membrane with blood. In the standard hemolysis assay, materials producing a 0 to $2 \% \mathrm{HR}$ are considered nonhemolytic materials, whereas materials producing a 2 to $5 \%$ HR or a $>$ $5 \%$ HR are classified as slightly hemolytic or hemolytic materials, respectively [25]. As shown in Table 2, the HR of the nerve repair membrane was $1.07 \%$, indicating that the nerve repair membranes were nonhemolytic materials and had good blood compatibility.

$\operatorname{HR}(\%)=\left(\mathrm{OD}_{\mathrm{a}}-\mathrm{OD}_{\mathrm{b}}\right) /\left(\mathrm{OD}_{\mathrm{c}}-\mathrm{OD}_{\mathrm{b}}\right) \times 100$

where $\mathrm{OD}_{\mathrm{a}}$ indicates the $\mathrm{OD}_{545}$ of the nerve repair membrane group, $\mathrm{OD}_{\mathrm{b}}$ indicates the $\mathrm{OD}_{545}$ of the negative control group, and $\mathrm{OD}_{\mathrm{c}}$ indicates the $\mathrm{OD}_{545}$ of the positive control group

\section{Functionality of the Nerve Repair Membrane In Vivo}

Claw-Spread Reflex Analysis The claw-spread reflex analysis indicated the functional nerve recovery following sciatic nerve injury, and the results ranged from grade A (normal function) to grade $\mathrm{C}$ (complete dysfunction). At 2 weeks after nerve anastomosis, the regenerative nerve fibers could not reach their targets, so the rats lost their claw-spread reflex. At 4, 8, and 12 weeks after surgery, the proportion of rats with grade $\mathrm{A}$ response gradually increased over time in both groups, demonstrating the gradual restoration of nerve function (Table 3). During the 12-week period, there were statistically significant differences in each grade between the treatment and nontreatment groups $(p<0.05)$.

Nerve Anastomosis Site Tissue Adhesion Evaluation The nerve repair membrane was used to wrap the right anastomosis site in the rats with transected sciatic nerves (Fig. 7a, b). At 2 weeks after surgery, the nerve repair membrane had not obviously 
Table 3 The rat number and proportion of each grade according to the claw-spread reflex test

\begin{tabular}{lllll}
\hline & Group & Grade A, $n(\%)$ & Grade B, $n(\%)$ & Grade C, $n(\%)$ \\
\hline \multirow{2}{*}{ 4 weeks $(N)$} & Treatment group* & $12(50.00)$ & $8(33.33)$ & $4(16.67)$ \\
& Nontreatment group & $7(29.17)$ & $10(41.67)$ & $7(29.17)$ \\
\multirow{2}{*}{12 weeks $(N)$ weeks $(N)$} & Treatment group* & $13(81.25)$ & $2(12.50)$ & $1(6.25)$ \\
& Nontreatment group & $9(56.25)$ & $4(25.00)$ & $3(18.75)$ \\
& Treatment group* & $7(87.50)$ & $1(12.50)$ & $0(0)$ \\
& Nontreatment group & $5(62.50)$ & $2(25.00)$ & $1(12.50)$ \\
\hline
\end{tabular}

$N=24$ rats at 4 weeks after surgery; $N=16$ rats at 8 weeks after surgery; $N=8$ rats at 12 weeks after surgery $* p<0.05$, versus the nontreatment group degraded, so the adhesion of the anastomosis site to surrounding tissues could not be observed. After the gradual degradation of the nerve repair membrane, the anastomosis site was observed at 4 weeks after surgery. At 8 weeks after surgery, the nerve repair membrane was completely degraded. Therefore, the anastomosis site adhesion was evaluated at 4,8 , and 12 weeks after surgery. At 12 weeks after surgery, there was no anastomosis site adhesion on the treatment side, but the anastomosis site was adhered to the surrounding tissues on the nontreatment side (Fig. 7c, d). As shown in Table 4, there were more rats at grade 0 in the treatment group than in the nontreatment group at each observation point. At the same time, compared to the rats in the nontreatment group, fewer rats in the treatment group were at grade 1 or grade 2 at each observation point. It is possible that the scoring of the anastomosis site adhesion at 4 weeks was affected by the residual membrane present in the treatment group, but the results from 8 and 12 weeks strongly proved that the nerve repair membrane could effectively inhibit anastomosis site tissue adhesion $(p$ $<0.05)$.

Electrophysiological Evaluation Electrophysiological assay was performed to evaluate the nerve functional recovery after nerve anastomosis. The MCV and CMAP amplitude of the normal sciatic nerve were $58.3 \pm 1.08 \mathrm{~m} / \mathrm{s}$ and $12.8 \pm 1.62$ $\mathrm{mV}$, respectively. At 2 weeks after surgery, the MCV and CMAP amplitude were not measured because nerve function had not recovered after such a short time. Therefore, the MCV and CMAP amplitudes of each group at 4, 8, and 12 weeks after surgery were detected (Fig. 8). At 4, 8, and 12 weeks, the
MCVs in the treatment group were $17.4 \pm 2.53,26.2 \pm 3.26$, and $32.4 \pm 2.84 \mathrm{~m} / \mathrm{s}$, respectively, while the MCVs in the nontreatment group were $15.6 \pm 3.15,19.3 \pm 4.78$, and 22.7 $\pm 3.96 \mathrm{~m} / \mathrm{s}$, respectively. At 4,8 , and 12 weeks, the CMAP amplitudes in the treatment group were $2.41 \pm 0.82,3.94 \pm$ 0.71 , and $6.85 \pm 1.31 \mathrm{mV}$, respectively, while the CMAP amplitudes in the nontreatment group were $2.21 \pm 0.92,2.35$ \pm 1.05 , and $4.74 \pm 1.67 \mathrm{mV}$, respectively. According to the electrophysiological results, the MCVs and CMAP amplitudes were significantly higher in the treatment group than in the nontreatment group at 8 and 12 weeks after surgery ( $p$ $<0.05$ ), while there was no difference between the 2 groups at 4 weeks. Although the MCVs and CMAP amplitudes of the treatment group did not return to normal during the observation period, nerve function was more significantly restored in the treatment group than in the nontreatment group.

Toluidine Blue Staining and Immunofluorescence Assay of the Axon and Myelin Sheath The distal segments near the anastomosis site were prepared for toluidine blue staining of the myelin sheath and immunofluorescence assay of the regenerated axon (NF200) and myelin sheath (S100) (Fig. 9). At 4,8 , and 12 weeks after surgery, the number of myelinated axons in each HPF $(\times 1000)$ was counted. The number of myelinated axons in the normal rat sciatic nerve was $311 \pm$ 16.6. As shown in Fig. 9b, the number of myelinated axons in the treatment group was $93.6 \pm 8.1,179.2 \pm 14.6$, and $275.2 \pm$ 17.3 at 4,8 , and 12 weeks, respectively, while the number was $73.3 \pm 8.3,141.1 \pm 16.9$, and $209.4 \pm 20.9$ in the nontreatment

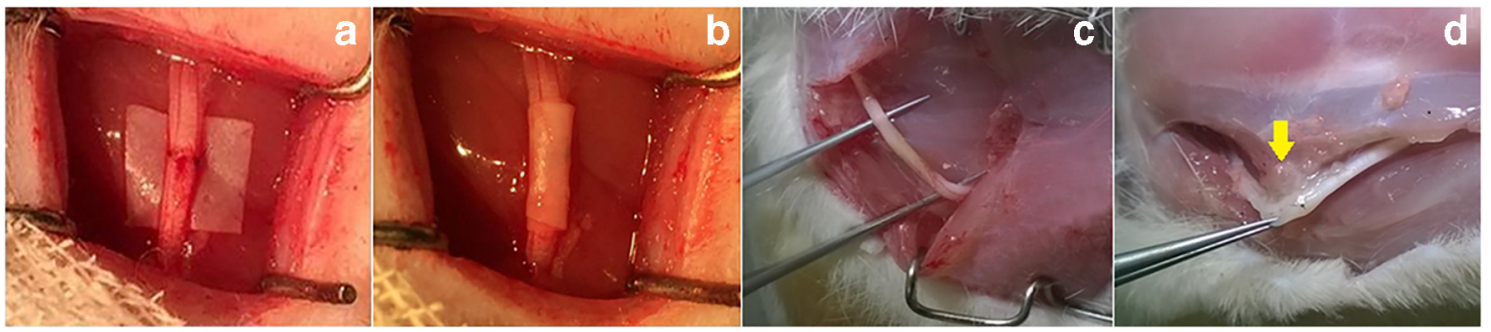

Fig. 7 The nerve repair membrane was used to wrap the right anastomosis site in a rat with a transected sciatic nerve (a, b). The tissue adhesion of the treatment side (c) and nontreatment side (d) at 12 weeks after surgery. The yellow arrowhead indicates anastomosis site adhesion 
Table 4 The number of rats of each grade according to the anastomosis site tissue adhesion evaluation

\begin{tabular}{lllll}
\hline & Group & Grade 0 $(n)$ & Grade 1 $(n)$ & Grade 2 $(n)$ \\
\hline \multirow{2}{*}{4 weeks $(N)$} & Treatment group* & 6 & 2 & 0 \\
& Nontreatment group & 0 & 5 & 3 \\
\multirow{3}{*}{12 weeks $(N)$} & Treatment group* & 6 & 2 & 0 \\
& Nontreatment group & 0 & 6 & 2 \\
& Treatment group* & 7 & 1 & 0 \\
& Nontreatment group & 0 & 5 & 3 \\
\hline
\end{tabular}

$N=8$ rats in each group

$* p<0.05$ versus the nontreatment group group at 4,8 , and 12 weeks, respectively. The results revealed that there were more myelinated axons on the treatment side than on the nontreatment side at 4,8 , or 12 weeks $(p<0.05)$.

Fig. 9c shows histomorphological micrographs of the normal rat sciatic nerve. At 2 weeks after surgery, a large number of myelin fragments and denatured nerve fibers were observed in the nerve sections from the treatment side and the nontreatment side (Fig. 9d, e).

At 4 weeks after surgery, the treatment side had more regenerated nerve fibers and fewer remaining denatured nerve fibers than the nontreatment side (Fig. 9d, e). The results showed that the number of myelinated nerve fibers on the treatment side was higher than that on the nontreatment side.

At 8 weeks after surgery, the number of myelinated nerve fibers on the treatment side was increased, and myelin became thickened (Fig. 9d, e). However, the number of myelinated nerve fibers on the nontreatment side was slightly increased.

At 12 weeks after surgery, the number of myelinated nerve fibers, the diameter of the nerve fibers, and the thickness of the myelin sheath on the treatment side were increased compared to the values on the nontreatment side (Fig. 9d, e).

\section{Discussion}

The repair of transected peripheral nerves requires the suturing of disconnected nerve ends [28]. However, direct suturing could lead to nerve anastomosis site adhesion to surrounding tissues, which impedes functional nerve recovery. Therefore, we have developed a novel xenogeneic, decellularized nervederived nerve repair membrane to prevent adhesion and promote nerve regeneration.

In this study, the decellularized nerve matrix derived from porcine sciatic nerves was verified to be effective in eliminating cellular components and therefore could avoid immune reactions after implantation of the tissue in the human body [15]. Meanwhile, decellularized nerve matrix preserved crucial ECM components, such as GAGs, collagens, LN, and FN [29]. The remaining GAGs in the decellularized nerve matrix could participate in the regulation of axonal growth [30]. Additionally, GAGs could partly promote the polymerization of collagens, which could contribute to the formation of the nerve repair membrane and support its mechanical properties $[31,32]$. As components of the basement membrane, collagens, LN, and FN could confer functionality to the nerve repair membrane to promote axonal outgrowth [33, 34]. For example, collagen conduits containing LN could improve the properties of hollow conduits in studies of nerve injury repair in animals [35].

The nano-RPLC-ESI-MS/MS system was used to evaluate the protein components of the nerve repair membrane, decellularized nerve matrix, and fresh nerve tissue in depth. There was no significant difference in protein mass between the nerve repair membrane and the decellularized nerve matrix, indicating that the process of generating the membrane preserved the ECM protein masses of the decellularized nerve matrix. Nevertheless, it is worth noting that collagen III, as a relatively abundant protein, could be detected in the proteomic
Fig. 8 Electrophysiological evaluation of functional nerve recovery. Histograms show the MCV (a) and CMAP amplitude (b) at 4, 8, and 12 weeks after nerve anastomosis. Data are expressed as the mean \pm S.D., * $p$ $<0.05$ a

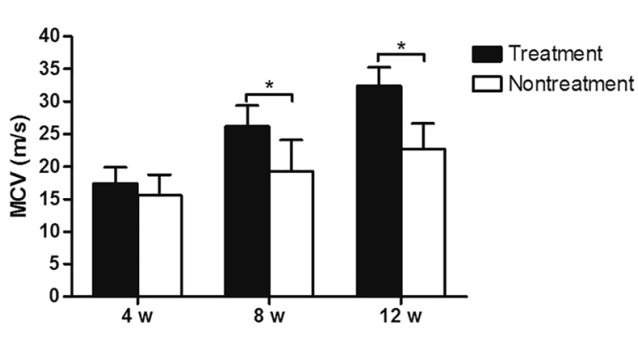

b

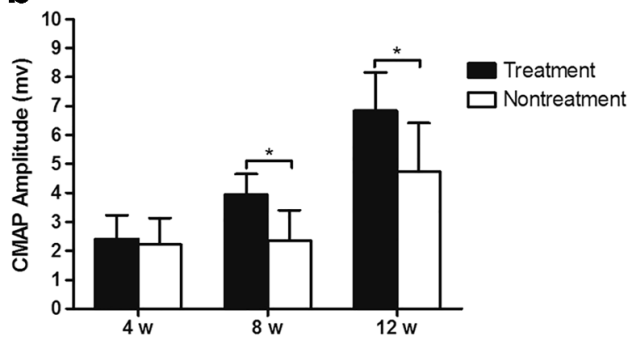


analysis but not in the Picrosirius red staining assay. Because the mass of collagen III was far less than the mass of collagen I according to the proteomic analysis, it was speculated that the green color (collagen III) might be covered by the red color (collagen I) of the Picrosirius red staining. Besides collagens, proteomic analysis also identified a large amount of the noncollagen protein myelin protein zero (MPZ), which is a major structural glycoprotein in peripheral myelin. Numerous studies have demonstrated that MPZ is important for myelin formation and neurite outgrowth after peripheral nerve injury [36-39]. Moreover, 1 cytokine, TGF- $\beta$, remained in the nerve repair membrane. As a member of a group of autocrine signaling molecules, TGF- $\beta$ can induce normal fibroblast transformation and regulate Schwann cell proliferation by activating the classical Smad and AKT pathways [40]. In addition, there are many other ECM proteins or degradation products that could be involved in intracellular signaling pathways, cell proliferation and differentiation, and vascularization [11, 18, 41-45]. For instance, collagens have chemotactic activity in endothelial cells and macrophages during the healing response [18]. The degradation fragments of LN and collagen IV, which contain X-Gly-X-X-Pro-Gly motifs, could bind to elastin binding protein on the surfaces of cells (such as various types of leukocytes) to trigger cellular activation [46]. However, the question of whether and how nerve repair membrane components regulate injured nerve repair by involving cell signaling pathways should be explored further.

The ability to support Schwann cell survival is a basic requirement for an ideal nerve repair material $[47,48]$. In this study, the results of the biocompatibility evaluation revealed that the nerve repair membrane could support Schwann cell growth and proliferation (Fig. 5 and Table 2). Some ECM proteins, including collagens and $\mathrm{FN}$, are necessary for Schwann cell growth and motility [16, 49, 50]. Therefore, the presence of these ECM proteins made the nerve repair membrane an ideal auxiliary biomaterial.

Here, we simulated conditions consistent with clinical application to use the nerve repair membrane to optimize the repair of transected peripheral nerves. In functional evaluation assays, the sciatic functional index is often calculated to assess the functional recovery of the motor nerve after nerve injury. However, rats might bite off their toes due to sensory dysfunction after sciatic nerve injury, which makes it difficult to record toe spread to calculate the sciatic functional index. This study used the claw-spread reflex test to evaluate the functional recovery of the rat sciatic nerve, which could not be affected by rat self-injury. Importantly, the claw-spread reflex test could reveal both motor and sensory nerve functional recovery, so it was a more appropriate indicator for nerve functional evaluation in our study. As shown in Table 3, the treatment sides produced the claw-spread reflex earlier than the nontreatment sides in rats, which indirectly showed that the nerve recovery time of the treatment group was significantly shorter than that of the nontreatment group. Additionally, the results of the electrophysiological and histological assays showed that the nerve function in the treatment sides recovered faster than that in the nontreatment sides. Notably, when the nerve anastomosis site was re-exposed, the nerve repair membrane was found to have significantly prevented adhesion between the anastomosis site and the surrounding tissues. After nerve injury, fibroblasts actively proliferate to cause adhesion between the anastomosis site and the surrounding tissues within 4 weeks. The nerve repair membrane acts as a mechanical barrier to prevent fibroblasts from invading into the anastomosis site. Four weeks later, although the nerve repair membrane (which included collagens, laminin, and fibronectin) had degraded gradually, the proliferation of fibroblasts also stopped gradually; thus, the anastomosis site did not adhere to the surrounding tissues. The nerve repair membrane could also preserve an isolated space for the anastomosis site to reduce bioactive molecule loss and enhance the effects of nutrients on the nerve. Therefore, the nerve repair membrane could effectively foster nerve regeneration in vivo.

Although the positive results of the in vitro and in vivo studies demonstrated that the nerve repair membrane had potential for clinical application, some important bioactive molecules were lost in the manufacturing process. This shortcoming could be compensated for by adding functional molecules, such as growth factors, or by improving the manufacturing technology used for the nerve repair membrane, thereby facilitating the reconstruction of the biological microenvironment to enhance nerve regeneration [51-54]. Additionally, based on the role of the nerve repair membrane in nerve anastomosis, it could be further used in other surgical operations. For instance, the nerve repair membrane could be used to wrap nerves after surgery for nerve compression syndromes to prevent nerve adhesion. Additionally, we could develop a novel xenogeneic, decellularized nerve-derived nerve repair conduit that could serve as an effective nerve graft in the future.

In conclusion, the xenogeneic, decellularized nervederived nerve repair membrane manufactured by the unique technique described in this study retained the main ECM components, and the in vitro and in vivo studies showed that the nerve repair membrane had good biocompatibility. Moreover, the use of the nerve repair membrane to repair the transected sciatic nerves of rats could effectively prevent surrounding tissues from invading the anastomosis site, reduce tissue adhesion, and enhance nerve regeneration. Additionally, clinical trials of this membrane have been conducted for the treatment of peripheral nerve disruption and nerve compression syndromes in 3 hospitals in China. 


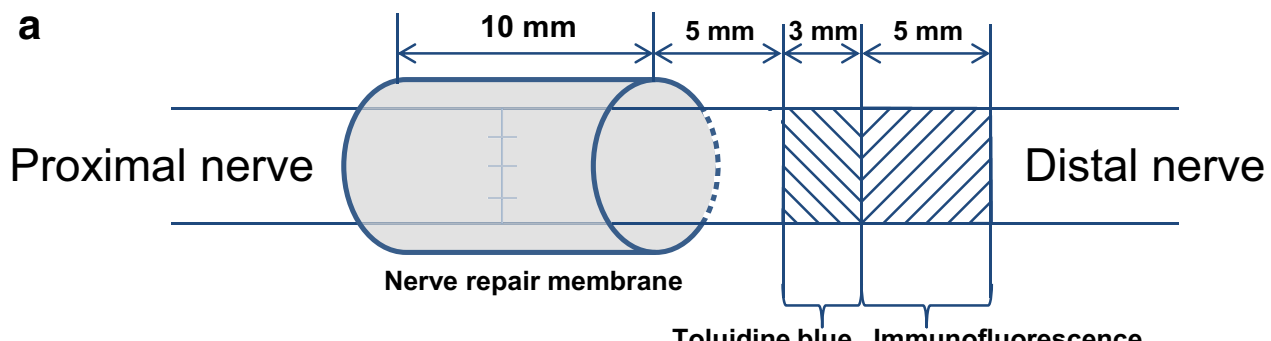

Toluidine blue Immunofluorescence

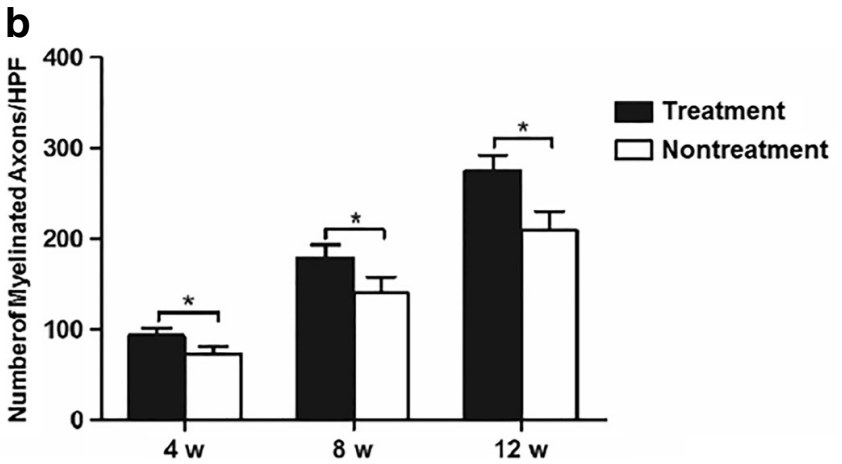

C

d

Treatment

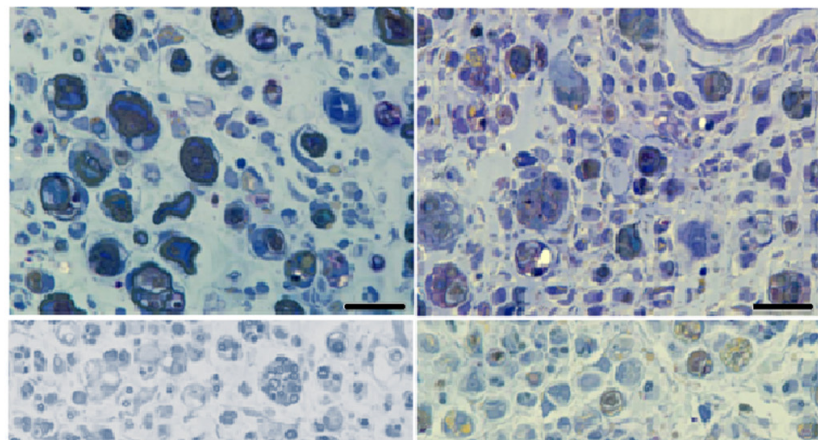

$4 \mathrm{~W}$

$W$

$12 \mathrm{~W}$

$8 \mathrm{~W}$
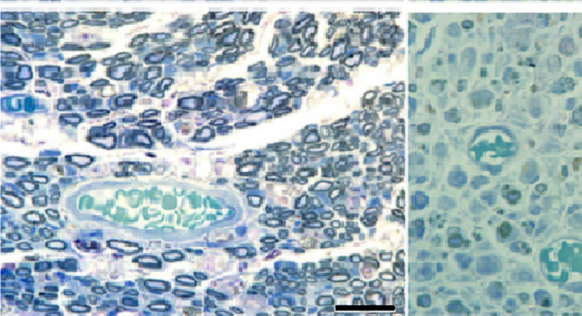

vive
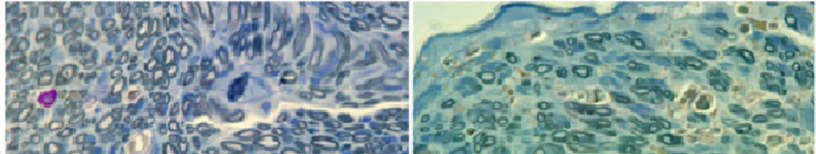
tom

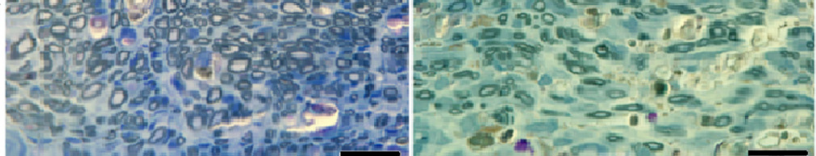

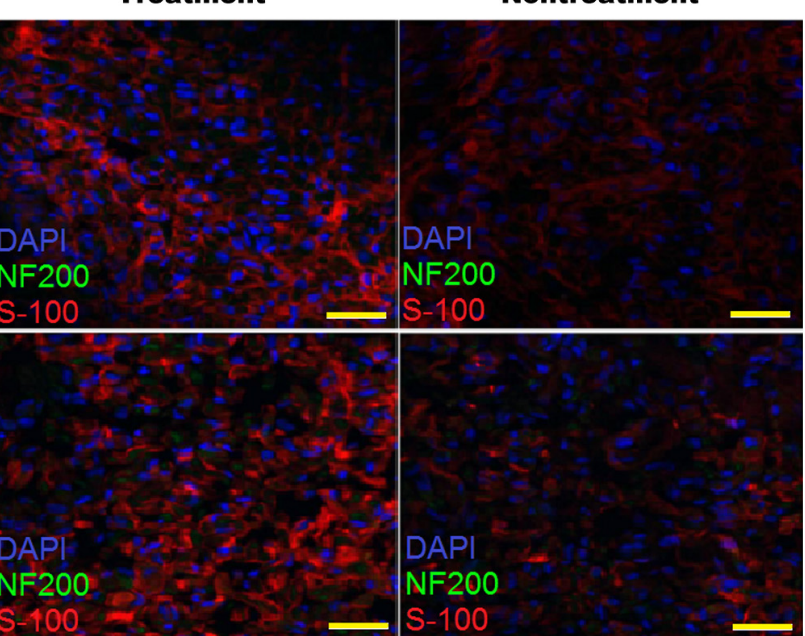

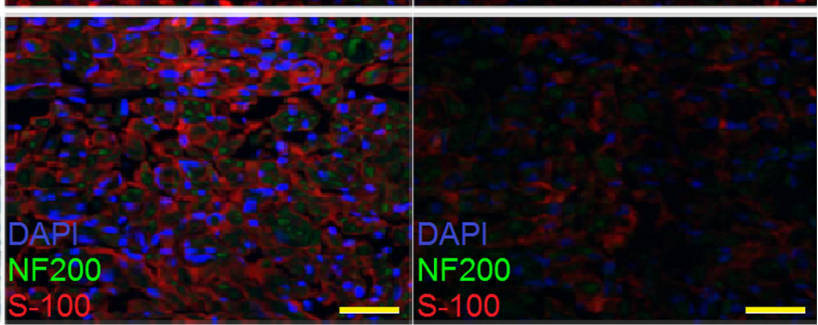

Normal nerve

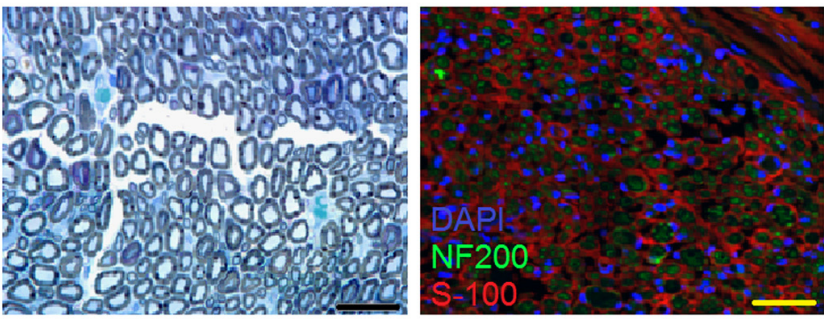

Treatment

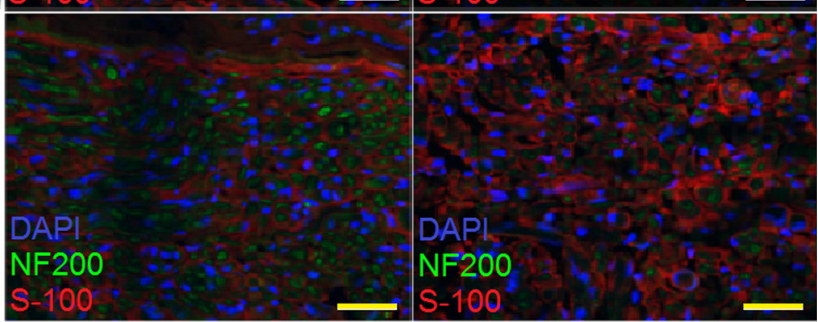


Fig. 9 Schematic diagram of the sampling locations of toluidine blue staining and immunofluorescence assay (a). Histogram of the myelinated axon numbers/HPF at 4,8 , and 12 weeks after surgery. Data are expressed as the mean \pm S.D., $* p<0.05$ (b). Toluidine blue staining and immunofluorescence micrographs of the normal rat sciatic nerve (c). Toluidine blue staining of the regenerated myelin sheath at $2,4,8$, and 12 weeks after surgery (d). Immunofluorescence staining of S-100 (red) and NF200 (green) in regenerated nerve fibers at 2, 4, 8, and 12 weeks after surgery; the cell nuclei were counterstained with DAPI (blue) (e). Scale bar $=20 \mu \mathrm{m}$

Acknowledgments This work was supported by the National Key Research and Development Program of China (Number 2017YFA0105802) and the National Natural Science Foundation of China (Number 81771351).

\section{Compliance with Ethical Standards}

The surgical procedure was performed strictly in accordance with the China Medical University guidelines for the ethical care of animals.

Required Author Forms Disclosure forms provided by the authors are available with the online version of this article.

\section{References}

1. Noble J, Munro CA, Prasad VS, Midha R. Analysis of upper and lower extremity peripheral nerve injuries in a population of patients with multiple injuries. J Trauma 1998;45:116-122.

2. Sachanandani NF, Pothula A, Tung TH. Nerve gaps. Plast Reconstr Surg 2014;133:313-319.

3. Trehan SK, Model Z, Lee SK. Nerve repair and nerve grafting. Hand Clin 2016;32:119-125.

4. Daly WT, Knight AM, Wang H, et al. Comparison and characterization of multiple biomaterial conduits for peripheral nerve repair. Biomaterials 2013;34:8630-8639.

5. Georgiou M, Bunting SC, Davies HA, et al. Engineered neural tissue for peripheral nerve repair. Biomaterials 2013;34:7335-7343.

6. Bockelmann J, Klinkhammer K, von Holst A, et al. Functionalization of electrospun poly( $\varepsilon$-caprolactone) fibers with the extracellular matrix-derived peptide GRGDS improves guidance of Schwann cell migration and axonal growth. Tissue Eng Part A 2011;17:475-486.

7. Moroder $\mathrm{P}$, Runge $\mathrm{MB}$, Wang $\mathrm{H}$, et al. Material properties and electrical stimulation regimens of polycaprolactone fumaratepolypyrrole scaffolds as potential conductive nerve conduits. Acta Biomater 2011;7:944-953.

8. Li R, Chen L, Fu J, Liu Z, Wang S, Pan Y. Promoting peripheral nerve regeneration with biodegradable poly (DL-lactic acid) films. Int J Clin Exp Pathol 2015;8:8057-8065.

9. Yao P, Li P, Jiang JJ, Li HY. Anastomotic stoma coated with chitosan film as a betamethasone dipropionate carrier for peripheral nerve regeneration. Neural Regen Res 2018;13:309-316.

10. Siemionow M, Uygur S, Ozturk C, Siemionow K. Techniques and materials for enhancement of peripheral nerve regeneration: a literature review. Microsurgery 2013;33:318-328.

11. Zhang Y, He Y, Bharadwaj S, et al. Tissue-specific extracellular matrix coatings for the promotion of cell proliferation and maintenance of cell phenotype. Biomaterials 2009;30:4021-4028.
12. Gilpin A, Yang Y. Decellularization strategies for regenerative medicine: from processing techniques to applications. Biomed Res Int 2017;2017:9831534.

13. Trevisan C, Maghin E, Dedja A, et al. Allogenic tissue-specific decellularized scaffolds promote long-term muscle innervation and functional recovery in a surgical diaphragmatic hernia model. Acta Biomater 2019;89:115-125.

14. Zilic L, Garner PE, Yu T, Roman S, Haycock JW, Wilshaw SP. An anatomical study of porcine peripheral nerve and its potential use in nerve tissue engineering. J Anat 2015;227:302-314.

15. Crapo PM, Gilbert TW, Badylak SF. An overview of tissue and whole organ decellularization processes. Biomaterials 2011;32: 3233-3243.

16. Zilic L, Wilshaw SP, Haycock JW. Decellularisation and histological characterisation of porcine peripheral nerves. Biotechnol Bioeng 2016;113:2041-2053.

17. Čunderlíková B, Filová B, Kajo K, et al. Extracellular matrix affects different aspects of cell behaviour potentially involved in response to aminolevulinic acid-based photoinactivation. J Photochem Photobiol B 2018;189:283-291.

18. Fu F, Zhu X, Qin Z, et al. Differential degradation rate and underlying mechanism of a collagen/chitosan complex in subcutis, spinal cord and brain tissues of rat. J Mater Sci Mater Med 2018;29: 35.

19. Zou JL, Liu S, Sun JH, et al. Peripheral nerve-derived matrix hydrogel promotes remyelination and inhibits synapse formation. Adv Funct Mater 2018;28:1705739.

20. Lin T, Liu S, Chen S, et al. Hydrogel derived from porcine decellularized nerve tissue as a promising biomaterial for repairing peripheral nerve defects. Acta Biomater 2018;73:326-338.

21. Zhao Q, Fang F, Wu C, et al. imFASP: an integrated approach combining in-situ filter-aided sample pretreatment with microwave-assisted protein digestion for fast and efficient proteome sample preparation. Anal Chim Acta 2016;912:58-64.

22. Stegemann H, Stalder K. Determination of hydroxyproline. Clin Chim Acta 1967;18:267-273.

23. Ignat'eva NY, Danilov NA, Averkiev SV, Obrezkova MV, Lunin VV, Sobol' EN. Determination of hydroxyproline in tissues and the evaluation of the collagen content of the tissues. J Anal Chem 2007;62:51-57.

24. Hou Y, Wang X, Yang J, Zhu R, Zhang Z, Li Y. Development and biocompatibility evaluation of biodegradable bacterial cellulose as a novel peripheral nerve scaffold. J Biomed Mater Res A 2018;106: 1288-1298.

25. Seo K, Kim D. pH-dependent hemolysis of biocompatible imidazole-grafted polyaspartamide derivatives. Acta Biomater 2010;6:2157-2164.

26. Jones G, Herbert A, Berry H, Edwards JH, Fisher J, Ingham E. Decellularization and characterization of porcine superflexor tendon: a potential anterior cruciate ligament replacement. Tissue Eng Part A 2017;23:124-134.

27. Wang B, Tedder ME, Perez CE, et al. Structural and biomechanical characterizations of porcine myocardial extracellular matrix. J Mater Sci Mater Med 2012;23:1835-1847.

28. Gu X, Ding F, Williams DF. Neural tissue engineering options for peripheral nerve regeneration. Biomaterials 2014;35:6143-6156.

29. Han GH, Peng J, Liu P, et al. Therapeutic strategies for peripheral nerve injury: decellularized nerve conduits and Schwann cell transplantation. Neural Regen Res 2019;14(8):1343-1351.

30. Lee JS, Shin J, Park HM, et al. Liver extracellular matrix providing dual functions of two-dimensional substrate coating and threedimensional injectable hydrogel platform for liver tissue engineering. Biomacromolecules 2014;15:206-218.

31. Stuart K, Panitch A. Influence of chondroitin sulfate on collagen gel structure and mechanical properties at physiologically relevant levels. Biopolymers 2008;89:841-851. 
32. Brightman AO, Rajwa BP, Sturgis JE, McCallister ME, Robinson JP, Voytik-Harbin SL. Time-lapse confocal reflection microscopy of collagen fibrillogenesis and extracellular matrix assembly in vitro. Biopolymers 2000;54:222-234.

33. Dalamagkas K, Tsintou M, Seifalian A. Advances in peripheral nervous system regenerative therapeutic strategies: a biomaterials approach. Mater Sci Eng C Mater Biol Appl 2016;65:425-432.

34. Chen ZL, Strickland S. Laminin gammal is critical for Schwann cell differentiation, axon myelination, and regeneration in the peripheral nerve. J Cell Biol 2003;163:889-899.

35. Matsumoto K, Ohnishi K, Sekine T, et al. Use of a newly developed artificial nerve conduit to assist peripheral nerve regeneration across a long gap in dogs. ASAIO J 2000;46:415-420.

36. Patzig J, Kusch K, Fledrich R, et al. Proteolipid protein modulates preservation of peripheral axons and premature death when myelin protein zero is lacking. Glia 2016;64:155-174.

37. da Silva JT, Santos FM, Giardini AC, et al. Neural mobilization promotes nerve regeneration by nerve growth factor and myelin protein zero increased after sciatic nerve injury. Growth Factors 2015;33:8-13.

38. Spiryda LB. Myelin protein zero and membrane adhesion. J Neurosci Res 1998;54:137-146.

39. Schneider-Schaulies J, von Brunn A, Schachner M. Recombinant peripheral myelin protein $\mathrm{P} 0$ confers both adhesion and neurite outgrowth-promoting properties. J Neurosci Res 1990;27:286-297.

40. Li M, Zhang P, Li H, Zhu Y, Cui S, Yao D. TGF- $\beta 1$ is critical for Wallerian degeneration after rat sciatic nerve injury. Neuroscience 2015;284:759-767.

41. Calve S, Odelberg SJ, Simon HG. A transitional extracellular matrix instructs cell behavior during muscle regeneration. Dev Biol 2010;344:259-271.

42. Vorotnikova E, McIntosh D, Dewilde A, et al. Extracellular matrixderived products modulate endothelial and progenitor cell migration and proliferation in vitro and stimulate regenerative healing in vivo. Matrix Biol 2010;29:690-700.

43. Cortiella J, Niles J, Cantu A, et al. Influence of acellular natural lung matrix on murine embryonic stem cell differentiation and tissue formation. Tissue Eng Part A 2010;16:2565-2580.
44. Ott HC, Clippinger B, Conrad C, et al. Regeneration and orthotopic transplantation of a bioartificial lung. Nat Med 2010;16:927-933.

45. Sellaro TL, Ravindra AK, Stolz DB, Badylak SF. Maintenance of hepatic sinusoidal endothelial cell phenotype in vitro using organspecific extracellular matrix scaffolds. Tissue Eng 2007;13:23012310.

46. Adair-Kirk TL, Senior RM. Fragments of extracellular matrix as mediators of inflammation. Int J Biochem Cell Biol 2008;40:1101-1110.

47. Fan BY, Zhou XH, Wang LN, et al. In vitro study of neural stem cells and activated Schwann cells cocultured on electrospinning polycaprolactone scaffolds. J Neurorestoratology 2017;5:155-165.

48. Kehoe S, Zhang XF, Boyd D. FDA approved guidance conduits and wraps for peripheral nerve injury: a review of materials and efficacy. Injury 2012;43:553-572.

49. Yang LM, Liu XL, Zhu QT, et al. Human peripheral nerve-derived scaffold for tissue-engineered nerve grafts: histology and biocompatibility analysis. J Biomed Mater Res B Appl Biomater 2011;96: 25-33.

50. Szynkaruk M, Kemp SW, Wood MD, Gordon T, Borschel GH. Experimental and clinical evidence for use of decellularized nerve allografts in peripheral nerve gap reconstruction. Tissue Eng Part B Rev 2013;19:83-96.

51. Houschyar KS, Momeni A, Pyles MN, et al. The role of current techniques and concepts in peripheral nerve repair. Plast Surg Int 2016;2016:4175293.

52. Zhuang $\mathrm{H}, \mathrm{Bu} \mathrm{S}$, Hua L, Darabi MA, Cao X, Xing M. Gelatinmethacrylamide gel loaded with microspheres to deliver GDNF in bilayer collagen conduit promoting sciatic nerve growth. Int J Nanomedicine 2016;11:1383-1394.

53. Qiu L, He B, Hu J, Zhu Z, Liu X, Zhu J. Cartilage oligomeric matrix protein angiopoeitin-1 provides benefits during nerve regeneration in vivo and in vitro. Ann Biomed Eng 2015;43:2924-2940.

54. Berrin ZA, Burcu D, Gamze A, Ömür GD. Melatonin and sciatic nerve injury repair: a current perspective. J Neurorestoratology 2018;6:49-60.

Publisher's Note Springer Nature remains neutral with regard to jurisdictional claims in published maps and institutional affiliations. 